Reviews in Mathematical Physics, Vol. 14, No. 5 (2002) 511-517

(C) World Scientific Publishing Company

\title{
ERRATUM TO "HADAMARD STATES, ADIABATIC VACUA AND THE CONSTRUCTION OF PHYSICAL STATES FOR SCALAR QUANTUM FIELDS ON CURVED SPACETIME" IN REV. MATH. PHYS. 8 (1996) 1091-1159
}

\author{
WOLFGANG JUNKER \\ Max-Planck-Institut für Gravitationsphysik, Albert-Einstein-Institut \\ Am Mühlenberg 1, D-14476 Golm, Germany \\ junker@aei-potsdam.mpg.de \\ Received 11 October 2001 \\ Revised 19 February 2002
}

In this erratum we want to correct or modify some of the original statements and proofs in "Hadamard States, Adiabatic Vacua and the Construction of Physical States for Scalar Quantum Fields on Curved Spacetime" in Rev. Math. Phys. 8 (1996) 1091-1159.

\section{E0. Introduction}

In the paper in question, we investigated Hadamard and adiabatic vacuum states for linear scalar field theories on curved spacetimes applying methods from microlocal analysis. In this erratum we want to correct or modify some of the original statements and proofs.

One of the main results of the paper was the derivation of a sufficient condition on certain pseudodifferential operators which guarantees that quasifree states of the Klein-Gordon field constructed from such operators are Hadamard states (Theorem 3.12). We have to include here the additional assumption that the spacetime under consideration has a compact Cauchy surface. Moreover, due to an error in the proof of the theorem the statement is slightly modified [Eqs. (74) and (75)], without however affecting its essence. The reason for these modifications and a precise restatement of Theorem 3.12 are presented in Sec. E1.

As an application of this theorem we had claimed in the paper that adiabatic vacuum states on Robertson-Walker spacetimes are Hadamard states (Theorem 3.24). This statement is wrong. In Sec. E2 we explain the origin of this error and prove the weaker statement that in the spatially compact case adiabatic vacua and Hadamard states generate unitarily equivalent GNS-representations. For a microlocal characterization of adiabatic vacuum states on arbitrary spacetimes and a clarification of their precise relation to Hadamard states in terms of wavefront sets we refer to [2]. 
Finally, in Sec. E3 we correct some further minor mistakes which have come to our attention since the publication of the paper.

\section{E1. Addendum to Theorem 3.12}

We have to add to the statement of Theorem 3.12 the assumption that the Cauchy surface $\Sigma$ is compact. We were implicitly using this e.g. in the proof of Lemma 3.13 when concluding that $W F\left(I_{2}\right) \equiv W F\left(K_{1}^{\Sigma}(1-\chi) \circ(1-\psi) K_{2}^{\Sigma}\right)=\emptyset$ from the fact that $K_{1}^{\Sigma}(1-\chi)$ and $(1-\psi) K_{2}^{\Sigma}$ are smooth. Since, however, these distributions are not properly supported, this statement implicitly involves an assumption about their global behaviour. One would have to supply the Riemannian manifold $(\Sigma, h)$ with asymptotic conditions to assure that our distributions are sufficiently well-behaved at infinity in order that no additional singularities ensue from their composition. By assuming that $\Sigma$ is compact we avoid this task (in fact, it makes Lemma 3.13 superfluous). Moreover, in part (v) of the proof of Theorem 3.12 we assumed that $\mathbf{1} \otimes P$ and $P \otimes \mathbf{1}$ with $P:=R-i I-n^{\alpha} \nabla_{\alpha}$ are "good" pseudodifferential operators possessing the pseudolocal property in order to conclude the $\subset$-relations in Eqs. (80) and (83). This, however, is not correct in two respects: Even when $R-i I$ is a pseudodifferential operator in $L_{1,0}^{1}$ w.r.t. the spatial variables and $n^{\alpha} \nabla_{\alpha}$ a differential operator in $L_{1,0}^{1}$ w.r.t. the time variable, then $R-i I-n^{\alpha} \nabla_{\alpha}$ is not a pseudodifferential operator in $L_{1,0}^{1}$ w.r.t. all variables unless $R-i I$ is a differential operator, too. Consider as an example $P:=-i\left(-\Delta+\mu^{2}\right)^{1 / 2}-\partial_{t}$ on $\mathbf{R}^{1+3}$. Its symbol $-i\left(|\boldsymbol{\xi}|^{2}+\mu^{2}\right)^{1 / 2}-i \tau$ is an element of $S_{0,0}^{1}\left(\mathbf{R}^{4} \times \mathbf{R}^{4}\right)$, but not of $S_{1,0}^{1}\left(\mathbf{R}^{4} \times \mathbf{R}^{4}\right)$ since taking derivatives w.r.t. $\xi_{i}$ does not lower the order w.r.t. the $\tau$-variable below 0 . The same reasoning shows that even if $P$ were in $L_{1,0}^{1}(\mathcal{M})$ then $\mathbf{1} \otimes P$ and $P \otimes \mathbf{1}$ would only be in $L_{0,0}^{1}(\mathcal{M} \times \mathcal{M})$ in general (unless $P$ were a differential operator). However, $L_{0,0}^{m}$ is a class of rather badly behaved pseudodifferential operators which are excluded in most of the statements of Chap. 2, in particular they do not possess the pseudolocal property in general. We claim that nevertheless Eqs. (80) and (83) are correct due to the particular properties of the Fourier integral operators $E^{+}$and $E^{-}$. For the precise proof we refer to Lemma 5.6 in [2], but the essential idea is that even when $P$ is only in $L_{0,0}^{1}(]-T, T[\times \Sigma)$ then one can find a pseudodifferential operator $X$ in $]-T, T[\times \Sigma$ such that char $X \cap N=\emptyset$ (where $N:=\left\{(x, \xi) ; g^{\mu \nu}(x) \xi_{\mu} \xi_{\nu}=0\right\}$ is the light cone in cotangent space) and $X P \in L_{1,0}^{1}(]-T, T[\times \Sigma)$ : Choose $X=o p \chi$, where $\chi$ is microlocally constructed as a real-valued function which is smooth on $T^{*}(]-T, T[\times \Sigma)$, zero for $|(\tau, \boldsymbol{\xi})| \leq 1 / 2$, homogeneous of degree zero for $|(\tau, \boldsymbol{\xi})| \geq 1$ such that $\chi(t, \mathbf{x} ; \tau, \boldsymbol{\xi})=0$ on a conic neighborhood of $\{\boldsymbol{\xi}=0\}$ and $\chi(t, \mathbf{x} ; \tau, \boldsymbol{\xi})=1$ outside a larger conic neighborhood of $\{\boldsymbol{\xi}=0\}$ that does not intersect the light cone. Due to the chosen support of $\chi$ the decrease in the $\tau$-variable of the symbol of $X P$ (and its derivatives w.r.t. $\xi_{i}$ ) can be estimated by that of the $\boldsymbol{\xi}$-variables and therefore $X P \in L_{1,0}^{1}(]-T, T[\times \Sigma)$. The expressions $(X P \otimes \mathbf{1}) E^{ \pm}$and $(\mathbf{1} \otimes X P) E^{ \pm}$can then be considered as compositions of the "good" pseudodifferential operator $X P$ and the Fourier integral operators 
$E^{ \pm}$which leave the wavefront sets of $E^{ \pm}$invariant and differ from $(P \otimes \mathbf{1}) E^{ \pm}$ and $(\mathbf{1} \otimes P) E^{ \pm}$, respectively, only by operators with smooth kernels due to $N \subset$ char $(X-1)$. A similar argument justifies Eq. (82) (see the proof of Lemma 5.6 in [2], here the additional assumption enters that $Q$ has a real-valued principal symbol). For the same reasons the operators $Q$ in the proof of Theorem 3.18, $Q_{n}$ in Eq. (122), and $Q_{1}, Q_{2}$ on p. 1153 have to be replaced by $i X Q, i X Q_{n}, i X Q_{1}$, and $i X Q_{2}$, respectively (the factor $i$ is introduced to make their principal symbols real-valued). This replacement changes Eq. (74) to

$$
Q\left(R-i I-n^{\alpha} \nabla_{\alpha}\right)=i X\left(\square_{g}+\mu^{2}+r_{1}(t)+r_{2}(t) n^{\alpha} \nabla_{\alpha}\right)
$$

and enlarges the characteristic set of $Q$ by char $X$. Since, however, char $X \cap N=\emptyset$ we still have char $Q \cap N_{-}^{\prime}=\emptyset$ (where $N_{-}^{\prime}:=\left\{(x, \xi) \in N ; \xi^{0}<0\right\}$ is the past light cone) and therefore none of the conclusions is affected by this change. Let us restate Theorem 3.12 including the mentioned changes (cf. Theorem 5.3 in [2]):

Theorem 3.12' . Let $I(t), R(t), S(t), C(t)$ be pseudodifferential operators on a compact $\left.\Sigma_{t}, t \in\right]-T, T[$ (satisfing the properties stated in Theorem 3.11 ) such that $I$ is elliptic, $S \in L^{-\infty}$ (from which follows that $C \in L^{0}$ is elliptic) and such that there exist pseudodifferential operators $Q$ and $X$ on $]-T, T[\times \Sigma$ which have the property

$$
Q\left(R-i I-n^{\alpha} \nabla_{\alpha}\right)=X\left(\square_{g}+\mu^{2}+r_{1}(t)+r_{2}(t) n^{\alpha} \nabla_{\alpha}\right)
$$

for some $r_{1}, r_{2} \in L^{-\infty}$, and $Q$ possesses a real-valued principal symbol $q$ with

$$
\operatorname{char} Q \cap N_{-}^{\prime}=\emptyset \text {. }
$$

Then the quasifree state given by (72) is an Hadamard state, i.e. the wavefront set of the two-point distribution $\Lambda_{\Sigma}^{(2)} \in \mathcal{D}^{\prime}(\mathcal{M} \times \mathcal{M})$ given by (73) is

$$
W F\left(\Lambda_{\Sigma}^{(2)}\right)=\left\{\left(x_{1}, \xi_{1} ; x_{2},-\xi_{2}\right) \in T^{*}(\mathcal{M} \times \mathcal{M}) \backslash\{0\} ;\left(x_{1}, \xi_{1}\right) \sim\left(x_{2}, \xi_{2}\right), \xi_{1}^{0} \geq 0\right\}
$$

(see Theorem 3.9).

\section{E2. Correction of Theorem 3.24}

The statement of Theorem 3.24 is wrong as it stands. The adiabatic vacuum states on the Robertson-Walker spacetimes are not of Hadamard type in general. This can be seen in the following way:

Suppose that the two-point functions $\Lambda_{n}^{(2)}$ and $\Lambda_{n+1}^{(2)}$ of two adiabatic vacuum states of order $n$ and $n+1$, respectively, are of Hadamard type. Then $\Lambda_{n}^{(2)}-\Lambda_{n+1}^{(2)} \in$ $\mathcal{C}^{\infty}(\mathcal{M} \times \mathcal{M})$, and consequently $\left.\Lambda_{n}^{(2)}\right|_{\Sigma_{t} \times \Sigma_{t}}-\left.\Lambda_{n+1}^{(2)}\right|_{\Sigma_{t} \times \Sigma_{t}} \in \mathcal{C}^{\infty}\left(\Sigma_{t} \times \Sigma_{t}\right)$ w.r.t. some Cauchy surface $\Sigma_{t}$ of a Robertson-Walker spacetime $\mathcal{M}$ (with flat spatial section, say). However, from the formula for the initial data of $\Lambda^{(2)}$ at the top of p. 1129 and Eq. (121) we note that $\left.\Lambda_{n}^{(2)}\right|_{\Sigma_{t} \times \Sigma_{t}}-\left.\Lambda_{n+1}^{(2)}\right|_{\Sigma_{t} \times \Sigma_{t}}$ is the kernel of a pseudodifferential operator with symbol

$$
\frac{1}{2}\left[\left(\Omega_{k}^{(n)}\right)^{-1}-\left(\Omega_{k}^{(n+1)}\right)^{-1}\right] .
$$


Introducing $\epsilon_{k}^{(n+1)}$ by

$$
\left(\Omega_{k}^{(n+1)}\right)^{2}=\left(\Omega_{k}^{(n)}\right)^{2}\left(1+\epsilon_{k}^{(n+1)}\right),
$$

noting from Lemma 3.2 of [4] that $\epsilon_{k}^{(n+1)} \in S^{-2 n-2}$ and remembering that $\omega_{k}:=$ $\left(k^{2} / a^{2}+m^{2}\right)^{1 / 2}$ is the leading term in the asymptotic expansion of $\Omega_{k}^{(n)}$ for any $n$ we find for (138) the principal symbol

$$
\frac{1}{4} \frac{\epsilon_{k}^{(n+1)}}{\omega_{k}} \in S^{-2 n-3} .
$$

In general, this is not a symbol in $S^{-\infty}$ (as can be explicitly checked from (115)), i.e. the corresponding pseudodifferential operator has not a smooth kernel, in contradiction to our assumption.

This contradiction to the statement of Theorem 3.24 has been noted by Hollands [1] when investigating the same question for Dirac fields, and implicitly also by Lindig [3] when calculating the singularity structure of the energymomentum tensor of the Klein-Gordon field in adiabatic vacuum states.

Let us now state and prove the correct relation between adiabatic vacua and Hadamard states. Due to the additional compactness assumption in Theorem $3.12^{\prime}$ (see Sec. E1) we restrict ourselves to the spatially compact case:

Theorem 3.24'. The adiabatic vacuum states of order $n \geq 0$ of the linear KleinGordon quantum field (100) of mass $\mu>0$ on the Robertson-Walker spacetimes (96) with $\kappa=+1$ (compact spatial sections) are unitarily equivalent to the Hadamard states.

Proof. The statement of the theorem is a consequence of Theorems 4.7 and 6.3 in [2] and Theorem 3.3 in [4]. For clarity's sake, however, we indicate the necessary corrections and modifications in the proof of Theorem 3.24. The main mistake was that we did not clearly distinguish between the solutions $T_{k}(t)$ of the differential equation (102) and their initial data $W_{k}^{(n)}(t)$, given by (114). They (and their first derivatives) are of course identical on the initial surface, say $\Sigma_{t_{0}}$, but not away from it. Therefore, in the expressions for $\Lambda_{n}^{(2)}$ on p. 1143, we can everywhere replace $T_{k}^{(n)}\left(t_{0}\right)$ and $\dot{T}_{k}^{(n)}\left(t_{0}\right)$ by $W_{k}^{(n)}\left(t_{0}\right)$ and $\dot{W}_{k}^{(n)}\left(t_{0}\right)$, respectively. But we assumed in the following that the Eqs. (119) are also valid for $t$ in an interval around $t_{0}$. This is not true in general, and Eq. (119) has to be replaced by

$$
\begin{aligned}
\left|W_{k}^{(n)}(t)\right|^{2} & =\left(a^{3} 2 \Omega_{k}^{(n)}(t)\right)^{-1}, \\
\frac{\dot{W}_{k}^{(n)}(t)}{W_{k}^{(n)}(t)} & =-\frac{3}{2} \frac{\dot{a}(t)}{a(t)}-\frac{1}{2} \frac{\dot{\Omega}_{k}^{(n)}(t)}{\Omega_{k}^{(n)}(t)}-i \Omega_{k}^{(n)}(t) .
\end{aligned}
$$

Then the definitions (121) and (122) of our operators $R_{n}, I_{n}$, and $Q_{n}$ remain correct (when replacing $\dot{T}_{k}^{(n)} / T_{k}^{(n)}$ in the 2 nd line of Eq. (122) by $\dot{W}_{k}^{(n)} / W_{k}^{(n)}$ and multiplying the r.h.s. of the definition of $Q_{n}$ in the 1 st line by $i X, X=o p \chi$, see the 
remark in Sec. E1) for $t$ in an interval around $t_{0}$. The statement of Lemma 3.26 still holds true uniformly in $t$ near $t_{0}$ when one replaces in (iii) $q(t ; \xi)$ by $i \chi q(t ; \xi)$ to take into account the redefinition of $Q_{n}$. (In the proof of Lemma 3.26 line 5 of p. 1146 is incorrect and has to be deleted. In the spatially compact case it follows from Theorem 2.28(b) that $D$ and the operators derived from it are pseudodifferential operators (cf. Lemma 6.5 in [2]).) However, the factorization of the Klein-Gordon operator now reads correctly

$$
\begin{aligned}
\left(-\partial_{t}-3 \frac{\dot{a}}{a}-\frac{\dot{W}_{k}^{(n)}}{W_{k}^{(n)}}\right)\left(\frac{\dot{W}_{k}^{(n)}}{W_{k}^{(n)}}-\partial_{t}\right) & =\partial_{t}^{2}+3 \frac{\dot{a}}{a} \partial_{t}-\frac{1}{W_{k}^{(n)}}\left(\ddot{W}_{k}^{(n)}+3 \frac{\dot{a}}{a} \dot{W}_{k}^{(n)}\right) \\
& =\square_{g}+\mu^{2}-\frac{1}{W_{k}^{(n)}}\left(\partial_{t}^{2}+3 \frac{\dot{a}}{a} \partial_{t}+\omega_{k}^{2}\right) W_{k}^{(n)} \\
& =\square_{g}+\mu^{2}+\left(\Omega_{k}^{(n+1)}\right)^{2}-\left(\Omega_{k}^{(n)}\right)^{2} \\
& =\square_{g}+\mu^{2}+\left(\Omega_{k}^{(n)}\right)^{2} \epsilon_{k}^{(n+1)}
\end{aligned}
$$

where we used (114), (115) and the definition (139) of $\epsilon_{k}^{(n+1)}$. From Lemma 3.2 in [4] it follows that $\left(\Omega_{k}^{(n)}\right)^{2} \epsilon_{k}^{(n+1)}=\mathcal{O}\left(k^{-2 n}\right)$ for $k \rightarrow \infty$, i.e. we have

$$
-Q_{n}\left(R_{n}-i I_{n}-\partial_{t}\right)=i X\left(\square_{g}+\mu^{2}\right) \bmod L^{-2 n}
$$

(uniformly for $t$ near $t_{0}$ ) and not $\bmod L^{-\infty}$ as we had claimed. Whereas $Q_{n}, R_{n}$, and $I_{n}$ are only defined by truncated asymptotic expansions $\bmod \mathcal{O}\left(k^{-2 n-1}\right)$ and $\mathcal{O}\left(k^{-2 n-2}\right)$, respectively, we can add up the asymptotic expansions defining these operators to infinite order (analogous to Lemma 2.3) obtaining operators $Q_{\infty}, R_{\infty}$, and $I_{\infty}$ (uniquely $\bmod L^{-\infty}$ ) which satisfy (140) for all $n \in \mathbf{N}$ and hence $\left(74^{\prime}\right)$, precisely as we did in the construction of Hadamard states in Sec. 3.7. Theorem 3.12 then implies that the states defined by $R_{\infty}$ and $I_{\infty}$ (after a possible redefinition $\bmod L^{-\infty}$ to make them satisfy the assumptions in Theorem 3.11) are Hadamard states. To show that the adiabatic vacua of order $n$ are unitarily equivalent to the Hadamard states we calculate the Bogoliubov transformation (116) between two such states and find, using (117), that

$$
|\beta(k)|=\left(2 a^{3}\right)^{-1}\left(I_{n}(k) I_{\infty}(k)\right)^{-1 / 2}\left|R_{\infty}(k)-R_{n}(k)-i\left(I_{\infty}(k)-I_{n}(k)\right)\right|
$$

where $I_{n}(k), I_{\infty}(k), R_{n}(k), R_{\infty}(k)$ denote the symbols of the corresponding operators (121) defining the states, hence $|\beta(k)|=\mathcal{O}\left(k^{-2 n-2}\right)$ for $k \rightarrow \infty$. Comparing with Theorem 4.5 of Lüders \& Roberts [4] we can conclude that for $\kappa=+1$ all adiabatic vacua are unitarily equivalent to Hadamard states.

We do not see any difficulty to extend this analysis to Robertson-Walker spacetimes with flat spatial sections $(\kappa=0)$ since in this case all operators are explicitly given as pseudodifferential operators with symbols that are constant in the spatial variable. To treat the case $\kappa=-1$, however, the use of a specially adapted pseudodifferential calculus on non-compact spaces seems to be necessary. 
Unfortunately, this correction of Theorem 3.24 leaves again open the question for the adiabatic vacuum of order 0 on the open universe, and we have to restrict the statement of Corollary 3.25 correspondingly:

Corollary 3.25'. All Hadamard states and adiabatic vacuum states of order $n \geq 0$ of the linear Klein-Gordon quantum field on the closed Robertson-Walker spacetimes $(\kappa=1)$ lie in the same local primary folium (quasiequivalence class) of states.

For the special spacetime manifolds chosen here, this already confirms our conjecture at the end of the paper, that, by truncating the asymptotic expansions of the operators, one obtains states that are locally quasiequivalent to Hadamard states. In the case of Dirac fields the same result has been established by Hollands [1]. In [2] Junker \& Schrohe extend the definition of adiabatic vacuum states from Robertson-Walker spacetimes to arbitrary globally hyperbolic spacetime manifolds by a generalized wavefront set condition (using the notion of the Sobolev wavefront set). With this definition Hadamard states are the adiabatic vacua of infinite order. A criterion analogous to (140) for quasifree states to be adiabatic in this extended sense can then be given, and the construction of adiabatic states can be carried out using the methods of Sec. 3.7.

\section{E3. Minor Corrections}

p. 1107: The last line should read:

$$
\begin{gathered}
W F(v)=-W F^{T}(v) \\
\text { where } W F^{T}(v):=\left\{\left(x_{1}, \xi_{1} ; x_{2}, \xi_{2}\right) \in T^{*}(X \times X) ;\left(x_{2}, \xi_{2} ; x_{1}, \xi_{1}\right) \in W F(v)\right\} .
\end{gathered}
$$

p. 1125: Eq. (71) should read $E_{F}:=-i \Lambda^{(2)}+\Delta_{A}$.

On line 8 from the bottom read timelike instead of lightlike.

p. 1127: On lines 13, 20, 22 read Eq. (73) instead of Eq. (2).

p. 1129: On lines 9 and 15 replace are lightlike connected by lie on a pair of lightlike geodesics that intersect on $\Sigma$.

p. 1153: Line 11 should read:

$$
\left.\Leftrightarrow\left(-r^{t}+i A^{1 / 2}+i s^{t}-\frac{1}{\sqrt{h}} \partial_{t} \sqrt{h}\right)\left(r^{t}-i A^{1 / 2}-i s^{t}-\partial_{t}\right)=P+r_{1}^{t} \quad \text { on }\right]-T, T[.
$$

\section{Acknowledgments}

I am grateful to Stefan Hollands for pointing out the contradiction to Theorem 3.24 and discussions about this issue, and to Rainer Verch for indicating the missing compactness assumption in Theorem 3.12. 


\section{References}

[1] S. Hollands, "The Hadamard condition for Dirac fields and adiabatic states on Robertson-Walker spacetimes", Comm. Math. Phys. 216 (2001) 635-661.

[2] W. Junker and E. Schrohe, "Adiabatic vacuum states on general spacetime manifolds: Definition, construction, and physical properties", to appear in Ann. H. Poincaré.

[3] J. Lindig, "Not all adiabatic vacua are physical states", Phys. Rev. D, 59:064011, 1999.

[4] C. Lüders and J. E. Roberts, "Local quasiequivalence and adiabatic vacuum states", Comm. Math. Phys. 134 (1990) 29-63. 